\title{
Investigation of a Functionalized Self-Assembling Nanopeptide Hydrogel Loaded With Adipose- Derived Mesenchymal Stem Cells To Promote Vascular Regeneration
}

\section{Zhanao Liu}

Qilu Hospital of Shandong University

Ailing Tian

Qilu Hospital of Shandong University

Xin Yi

Qilu Hospital of Shandong University

\section{Lufeng Fan}

Qilu Hospital of Shandong University

\section{Wenchong Shao}

Qilu Hospital of Shandong University

\section{Han Wu}

Qilu Hospital of Shandong University

Nianfeng Sun ( $\sim$ sunnianfeng@126.com )

Qilu Hospital of Shandong University https://orcid.org/0000-0002-1997-9832

\section{Research article}

Keywords: Adipose derived mesenchymal stem cells, self-assembling peptide, hydrogel, cell transplantation, ischemic disease

Posted Date: November 10th, 2020

DOl: https://doi.org/10.21203/rs.3.rs-72114/v1

License: (c) (1) This work is licensed under a Creative Commons Attribution 4.0 International License. Read Full License 


\section{Abstract}

Background: To investigate the transplantation of a functionalized self-assembling nanopeptide hydrogel loaded with adipose-derived mesenchymal stem cells (AD-MSCs) into a rabbit hindlimb ischemia model and to evaluate its effect in promoting vascular regeneration in ischemic tissues.

Methods: Functionalized self-assembling nanopeptide hydrogels were synthesized, and the physical and chemical properties of the hydrogels were observed by electron microscopy. Primary AD-MSCs were isolated, cultured, immunophenotyped, induced to differentiate, and verified. The self-assembling nanopeptide hydrogel was combined with mesenchymal stem cells for the three-dimensional culture of AD-MSCs, and the growth characteristics were investigated. Animal models were injected with AD-MSCloaded self-assembled peptide hydrogel, and the therapeutic effects on arterial ischemia were analyzed.

Results: The pore size of the functionalized self-assembling nanopeptide hydrogel was suitable for cell growth. Stem cells had a tendency for migration, differentiation, and angiogenesis in three-dimensional culture. The experimental results of transplantation into the rabbit hindlimb ischemia model showed that the functionalized self-assembling nanopeptide hydrogel loaded with AD-MSCs had better efficacy than AD-MSC transplantation alone.

Conclusion: Functionalized self-assembling nanopeptide hydrogels can be used as scaffold materials for three-dimensional culture of AD-MSCs. Functionalized self-assembling peptide hydrogels combined with AD-MSCs have better therapeutic effects than traditional stem cell therapies and can promote vascular regeneration.

\section{Background}

Bone marrow mesenchymal stem cells have limited practical applications because they are difficult to obtain on a large scale ${ }^{[1]}$. The acquisition of adipose-derived mesenchymal stem cells (AD-MSCs) is more convenient and more suitable for large-scale expansion. AD-MSCs have low immunogenicity and multi-directional differentiation potential. AD-MSCs have been successfully differentiated into osteoblasts, chondrocytes, adipocytes, cardiomyocytes, endothelial cells, and neurons ${ }^{[2-6]}$. Therefore, AD-MSCs, as a new type of seed cell, have good application prospects in medicine.

The biological materials selected in the field of tissue engineering must be free of toxicity and side effects, be absorbable and degradable, and have good compatibility with seed cells and the organism receiving transplantation ${ }^{[7]}$. The combined transplantation of self-assembling nanopeptide hydrogel and stem cells may be able to buffer sudden changes in the living environment of stem cells. The growth, proliferation, and migration of stem cells in a three-dimensional culture scaffold may achieve a slow release and long-lasting effect on local lesion tissues. Therefore, the use of self-assembling nanopeptide hydrogel scaffolds loaded with stem cells could be an improvement over the traditional simple stem cell suspension injection method and should have good application prospects in the field of stem cell transplantation. 
In recent years, there have been many studies related to stem cell transplantation for the treatment of

ischemic diseases ${ }^{[8-10]}$. Transplantation of bone marrow mesenchymal stem cells or other types of stem cells into tissues and organs with ischemia caused by arterial occlusion could produce "therapeutic angiogenesis", thus promoting the formation of more collateral circulations and achieving the goal of improving the blood flow to ischemic tissue ${ }^{[11,12]}$.

In this study, a functionalized self-assembling nanopeptide hydrogel was prepared and combined with AD-MSCs for transplantation into a hindlimb ischemia model in rabbits to study its effect of promoting vascular regeneration in ischemic tissues.

\section{Methods}

All animal experiments have been approved by the Research Ethics Committee of Shandong University(Approval number: KYLL-2015(KS)-200) and National Institutes of Health (NIH) Guidelines for the Care and Use of Laboratory. Pure-bred male New Zealand white rabbits weighing between $2.5 \mathrm{~kg}$ and $3.0 \mathrm{~kg}$ were purchased from the Animal Testing Center of Shandong University. All the $15 \mathrm{New}$ Zealand rabbits were healthy and had similar hind limb nutritional status and arterial pulsation. Fifteen rabbits were randomly divided into three groups: Experimental Group, Negative Control Group and positive control group.

\section{Culture and differentiation induction of AD-MSCs 1.1 Primary culture of AD-MSCs}

Fresh adipose tissue was cut into pieces and digested with type I collagenase. After centrifugation, resuspension, and filtration, the obtained cells were added to DMEM/F12 complete culture medium, and the resulting $\mathrm{P}_{0}$ culture was placed in a $37^{\circ} \mathrm{C}, 5 \% \mathrm{CO}_{2}$ incubator with saturated humidity. Cell growth and cell morphology were observed daily under an inverted phase-contrast microscope. The $\mathrm{P}_{0}$ AD-MSCs were digested with trypsin-EDTA, and the digestion was ended when the cells became oval and the intercellular space became larger. The adherent cells were suspended, added again to DMEM/F12 complete culture medium, and inoculated into a new culture bottle at a 1:3 ratio with complete culture medium with $10 \%$ fetal bovine serum. This became the $\mathrm{P}_{1}$ culture. Cells were later passaged using the same method.

\subsection{Immunophenotype identification of AD-MSCs}

AD-MSCs $\left(P_{3}\right)$ at $80 \%$ confluence were digested with $0.25 \%$ trypsin-EDTA. Cells were resuspended in phosphate-buffered saline (PBS) solution and distributed into six $0.5 \mathrm{ml}$ Eppendorf tubes. Phycoerythrin (PE)-labeled rat anti-human cluster of differentiation (CD) 29, CD31, CD34, CD44, and human leukocyte antigen-DR isotype (HLA-DR) flow cytometry antibodies and immunoglobulin G1 (IgG1) isotype (negative control) were added to separate Eppendorf tubes. After mixing by pipetting, the tubes that had antibodies were incubated for 20 minutes in the dark and at room temperature, followed by a PBS wash, 
resuspension of the cell pellet in $0.5 \mathrm{ml}$ PBS, and detection of the immunophenotypes of AD-MSCs in a flow cytometer.

\subsection{Drawing the growth curve of AD-MSCs}

AD-MSCs $\left(\mathrm{P}_{3}\right)$ at $80 \%$ confluence was digested with $0.25 \%$ trypsin-EDTA, the density of AD-MSCs in the cell suspension was adjusted to $3 \times 10^{5}$ cells $/ \mathrm{ml}$, and the cell suspension was added to six wells of a 96well plate at $100 \mu \mathrm{l} /$ well that were included in the experimental group, while one well received $100 \mu \mathrm{l}$ of complete medium as the blank control. A total of eight 96-well plates were inoculated. One 96-well plate was taken every 24 hours, $10 \mu \mathrm{l}$ 0.5\% 3-(4,5-dimethylthiazol-2-YI)-2,5-diphenyltetrazolium bromide (MTT) was added to each well, and the plate was incubated again for 4 hours and then removed again. DMSO was then added to the plate at $100 \mu \mathrm{l} /$ well, and a microplate reader was used to measure the optical density (OD) value of each well at $490 \mathrm{~nm}$. The wells that only had medium were the blank control group, which was used to adjust the baseline, and the data of six experimental wells were recorded and the average value was taken. The rest of the 96-well plates remained in the cell culture incubator, and the medium was changed every 2-3 days. One 96-well plate was taken every 24 hours to repeat the above steps. After recording the data for 8 days, the cell growth curve was drawn based on the average OD value obtained every day, and the shape of the cell growth curve was observed.

\subsection{Determination of AD-MSC cell cycle}

AD-MSCs $\left(\mathrm{P}_{3}\right)$ at $80 \%$ confluence were digested with $0.25 \%$ trypsin-EDTA. After centrifugation, the supernatant was aspirated, and $1 \mathrm{ml}$ of $70 \%$ ethanol stored at $4{ }^{\circ} \mathrm{C}$ overnight was added to resuspend the cell pellet by pipetting. The cells were fixed in a refrigerator at $4{ }^{\circ} \mathrm{C}$ overnight. On the second day, the cells were centrifuged for 5 minutes, the supernatant was aspirated, and the cells were resuspended by adding PBS at $4{ }^{\circ} \mathrm{C}$. After centrifugation again, the cell cycle detection kit was used to stain the fixed cells, and the cells were detected by flow cytometry to analyze the cell cycle of AD-MSCs.

\subsection{Induction of osteogenic differentiation of AD-MSCs}

AD-MSCs $\left(\mathrm{P}_{3}\right)$ at $80 \%$ confluence were digested with $0.25 \%$ trypsin-EDTA and seeded at a density of $1.5 \times$ $10^{3} \mathrm{cells} / \mathrm{ml}$ into a six-well plate. After adding the cell suspension, the six-well plate was placed in a cell incubator. After 24 hours, the six-well plate was removed. After observing that most of the cells became adherent under an inverted phase-contrast microscope, the culture medium was aspirated and replaced with $2.5 \mathrm{ml}$ of osteogenic induction medium. The six-well plate was placed in the cell incubator to continue the culture. The osteogenic induction medium was routinely replaced every $2-3$ days. After 3 weeks of induction, AD-MSCs were stained with alizarin red S staining solution to observe the formation of calcium nodules.

\subsection{Induction of adipogenic differentiation of AD-MSCs}

AD-MSCs $\left(\mathrm{P}_{3}\right)$ at $80 \%$ confluence were digested with $0.25 \%$ trypsin-EDTA and seeded at a density of $1 \times$ $10^{4}$ cells/ml into a six-well plate with $2.5 \mathrm{ml}$ of cell suspension per well. The plate was placed in a cell 
incubator, and the medium was routinely replaced every 2-3 days. After AD-MSCs reached 100\% confluence, $2.5 \mathrm{ml}$ of adipogenic induction medium A was added. After 3 more days of culture, the adipogenic induction medium $A$ was discarded and replaced with adipogenic induction medium $B$. After 24 hours of culture, the adipogenic induction medium $B$ was replaced with the induction medium $A$, followed by incubation in a cell incubator for 3 days. After 12 days of repeating the above medium switches, media A and B each had been replaced three times. After observing obvious lipid droplets under an inverted phase-contrast microscope, oil red 0 staining was used to observe the results of adipogenic differentiation.

\subsection{Induction of endothelial differentiation of AD-MSCs}

The digested $\mathrm{P}_{3}$ suspension was added to a $15-\mathrm{ml}$ sterile centrifuge tube and centrifuged at $1000 \mathrm{r} / \mathrm{min}$ for 5 minutes. The upper layer in the centrifuge tube was discarded, the cells were washed with $1 \times$ PBS and centrifuged again, and then the endothelial cell support solution $100 \%$ EGM2-MV was added to suspend the cells. After resuspension, the cells were added to a culture flask. The morphological changes of the cells were continuously observed every day using an inverted phase-contrast microscope. The culture medium was replaced every 3 days, and the cell growth was closely observed. After 4 weeks, CD31-PE was used to label cells that were induced and cells that were not induced to undergo differentiation. Immunophenotypes were identified by flow cytometry.

\section{Preparation of functionalized self-assembling nanopeptide hydrogel and three-dimensional culture of combined AD-MSCs}

\subsection{Preparation of peptide solution and detection by atomic-force microscopy}

The three peptide powders were dissolved in a $10 \%$ glucose solution to obtain three kinds of peptide solutions, each having a mass concentration of $10 \mathrm{~g} / \mathrm{L}(1 \%)$. The peptide was completely dissolved by ultrasound treatment. The aqueous solutions of RADA16-I polypeptide, KLT functionalized polypeptide, and arginylglycylaspartic acid (RGD) functional polypeptide were mixed at a volume ratio of 2:1:1 and mixed well by ultrasound to obtain a functionalized self-assembling polypeptide solution (RADA/KLT/RGD). After diluting 100-fold and mixing by ultrasonic treatment, $10 \mu \mathrm{l}$ of the sample was dropped onto a freshly peeled mica sheet, dried, and observed under an atomic-force microscope.

2.2 Preparation of functionalized self-assembling nanopeptide hydrogels and observation of the microstructure of the gels by scanning electron microscopy

A Transwell chamber was used to complete the self-assembly of the peptide solution. The chamber was placed in a 24-well culture plate with PBS and incubated at $37^{\circ} \mathrm{C}$ overnight, in order to permeate the basement membrane. Then, $100 \mu \mathrm{L}$ of the functionalized self-assembling peptide solution (RADA/KLT/RGD) was added into the chamber, the chamber was removed after gelation, and the 
basement membrane was cut off to remove the hydrogel, which was fixed in $2.5 \%$ glutaraldehyde and dehydrated to prepare samples for scanning electron microscopy.

\subsection{Three-dimensional culture of functionalized self- assembling nanopeptide hydrogel combined with AD-MSCs}

After digesting the $\mathrm{P}_{3}$ AD-MSCs, the cell suspension was adjusted to $1 \times 10^{6} / \mathrm{ml}$, and $100 \mu \mathrm{L}$ of the suspension was inoculated into the hydrogel soaked with the culture medium. After overnight incubation, the hydrogel was rinsed. After 5 days of culture, the morphology and migration status of AD-MSCs were observed under an inverted phase-contrast microscope. The hydrogel in the chamber was then digested with digestive enzymes and quickly stained with acridine orange/ethidium bromide (AO/EB) for 30 seconds, followed by observation under a fluorescence microscope to exam the conditions of cell growth and apoptosis and to count cells.

\section{Establishment of a rabbit hindlimb ischemia model and stem cell transplantation}

\subsection{Rabbit hindlimb ischemia model and angiography}

Each rabbit was anesthetized with $3 \%$ sodium pentobarbital solution. After disinfection, the operation area was covered with sterile drapes. The skin from $1 \mathrm{~cm}$ above the midpoint of the inguinal ligament to the knee joint line was longitudinally cut, the femoral artery was dissected, the arterial collaterals were ligated separately from the inguinal ligament to the knee, and the femoral artery in this area was resected. A longitudinal incision was made in the midline of the neck, slightly leading towards the right side, to cut open the skin and subcutaneous tissue. A section of the common carotid artery approximately $2 \mathrm{~cm}$ long was dissected and placed with an arterial sheath entering the level of the aortic arch. Then $10 \mathrm{ml}$ of contrast agent was quickly injected. Digital angiography of bilateral hindlimbs was conducted for at least 10 seconds to observe the arteries of the hindlimbs of rabbits after operation.

\subsection{Transplantation of functionalized self-assembled nanopeptide hydrogel-loaded AD-MSCs into the rabbit model of hindlimb ischemia}

At 48 hours after operation on rabbit hindlimbs, rabbits in the experimental group (group A) were transplanted with the self-assembled polypeptide hydrogel combined with AD-MSCs at multiple sites in the right gastrocnemius muscle. Rabbits in the positive control group (group B) received multi-point injections of a suspension of PBS-washed AD-MSCs at the same location and with the same number of cells. Rabbits in the negative control group (group C) were injected with the same amount of PBS at the same sites. Limb movements, skin color, and the presence or absence of skin ulcers, loss of toenails, and limb gangrene in the hindlimbs on both the operation side and the healthy side were observed daily, and blood pressure, skin temperature, and arterial pulsation were measured and recorded. 


\subsection{Histological examination after stem cell transplantation}

At 30 days after AD-MSC transplantation, muscle samples were taken from the same sites of both hindlimbs (gastrocnemius muscles) and sectioned for hematoxylin-eosin (HE) staining. We observed whether the muscle tissues had necrosis, whether the muscle fibers were arranged neatly, and whether the muscle space was widened under the microscope. Immunofluorescence staining of factor VIII was conducted on the abovementioned frozen sections. The sections were fixed in $4 \%$ paraformaldehyde solution, dried, permeabilized, and blocked in $5 \%$ goat serum blocking solution. The sections were then added to factor VIII primary antibody diluted 1:100 and FITC-labeled secondary antibody in drops and incubated in a humidified box at room temperature. The slides were sealed after drying, and the number of cells positive for immunofluorescence staining in a single field were counted under a fluorescence microscope.

\section{Statistical analysis}

The recorded experimental data were processed using SPSS18.0. The data are expressed as the mean \pm standard deviation and were analyzed using pairwise comparisons in analysis of variance (ANOVA) within SPSS. The test standard for statistical significance was $\mathrm{P}<0.05$.

\section{Results}

\section{Culture and induction of AD-MSCs}

\subsection{Morphological observation of AD-MSCs}

Twenty-four hours after inoculation of isolated AD-MSCs, cells were observed under an inverted phasecontrast microscope, and it was found that a smaller number of short-spindle-shaped cells were adherent (Fig. 1-1A). After 2 days, observation showed that the initial adherent cells grew slowly. The cell morphology at 6 days is shown in Fig. 1-1B. After 6-10 days of culture, the growth rate of adherent cells began to speed up, and cells started to show long-spindle, fibroblast-like, and school-of-fish-like morphology (Fig. 1-1C). During the next 10 passages, the cells maintained their fibroblast-like morphology and high proliferative activity, and the morphology did not significantly change (Fig. 1-1D).

\subsection{Combined immunophenotyping of AD-MSCs}

Flow cytometry was used to detect that the expression of multiple markers characteristic of AD-MSCs. CD29 and CD44 were positive, but the endothelial cell marker CD31, the hematopoietic marker CD34, and the fibroblast marker HLA-DR were negative, proving that the primary cultured cells were AD-MSCs (Fig. 1-2).

\subsection{Growth curve drawing and cell cycle determination for AD-MSCs}

The OD values of eight 96-well plates inoculated with AD-MSCs were measured by a microplate reader after using the MTT method, and the cell growth curve was drawn using the data (mean values) over 8 days. It was found that the growth curve of AD-MSCs was close to an S shape. The growth of stem cells was slower in days 1-4, while the growth rate became faster and cells entered the exponential growth stage after the 4th day. After 7 days, the cell density had become higher, and cells entered a plateau phase in which the growth rate slowed down. It is consistent with the general growth pattern of cells 
(Fig. 1-3A). Flow cytometry analysis of the cell cycle showed that approximately $77 \%$ of AD-MSCs were in the G0/G1 phase (quiescent phase) and 23\% of AD-MSCs were in the $S+G 2 / M$ phase (proliferation phase, Fig. 1-3B).

\subsection{Induction of osteogenic and adipogenic differentiation}

AD-MSCs were put through 3 weeks of osteogenic differentiation induction, and alizarin red S staining showed the formation of dark-brown mineralized nodules (Figs. 1-4A, 4C). During the induction of ADMSCs to differentiate into adipocytes, the trend of differentiation of AD-MSCs into adipocytes could be seen approximately 10 days after induction. The cytoplasm began to fill with fat particles, and oil red 0 staining of induced AD-MSCs revealed positive staining (Figs. 1-4B, 4D). By demonstrating their multidirectional differentiation potential, these data further show that the isolated and cultured primary cells were AD-MSCs.

1.5 Inductions of endothelial differentiation of AD-MSCs and flow cytometry identification After AD-MSCs were induced to endothelial differentiation, it was found that the spindle cells became shorter, appeared to show polygonal or triangular-like changes, and the cells showed a paving-stone-like overall growth status. There was no obvious morphological change in the control group (Fig. 1-5A). Flowcytometric immunophenotyping was performed on the stem cells after endothelial differentiation induction, and the cells were labeled and loaded into the flow cytometer. We found that the endotheliumlabeling antibody CD31 changed from negative to positive, while the control group still had negative CD31 results in flow cytometry (Figs. 1-5B, 5C).

\section{Preparation of functionalized self-assembling nanopeptide hydrogels and three-dimensional culture of combined fat mesenchymal stem cells}

2.1 Preparation of peptide solution and detection by atomic-force microscopy

The peptide powder was dissolved in $10 \%$ glucose to form a peptide solution. The peptide solution was thick and turbid. After sonication with an ultrasound probe, the peptide solution changed from a thick, turbid solution to a clear solution, indicating that the peptide was dissolved and mixed well. Atomic-force microscopy revealed nanofiber-like structures made of the peptides. The length and thickness of the peptide nanofibers were relatively uniform, and the fiber diameter was 10-20 nm (Fig. 2-1A).

2.2 Preparation of self-assembled nanopeptide hydrogel

Hydrogel was obtained after the self-assembly of the RADA/KLT/RGD polypeptide solution was induced by PBS. It was transparent and had a solid hydrogel-like property, and its water content was extremely rich (Fig. 2-1B, 1C). Scanning electron microscopy showed that the polypeptides aggregated into polymer fibers and formed a network-like fiber structure with a pore diameter of approximately 10-50 $\mu \mathrm{m}$. Since most eukaryotic cells have a diameter of 5-30 $\mu \mathrm{m}$, the hydrogel was suitable for cell adhesion, migration, and growth inside the hydrogel, providing a true 3D culture environment (Fig. 2-1D).

2.3 In vitro experiments combining cells with self-assembling peptide hydrogels When AD-MSCs were seeded on a self-assembling nanopeptide hydrogel, the cells were evenly distributed and had a slightly different morphology, but the cells were in a good state of extension and were tightly connected (Fig. <link rid="fig9">2</link>-2).

2.4 Cell compatibility of self-assembling peptide hydrogel 
AD-MSCs were cultured in the self-assembling nanopeptide hydrogel. Cells grew well in the hydrogel, were spread out, migrated, and were interconnected. The cell suspension was stained with acridine orange (AO)/ethidium bromide (EB) for 30 seconds and observed under a fluorescence microscope. Four fields were randomly selected under a 10x objective lens for cell counting (Fig. 2-3). It was found that apoptotic cells made up fewer than $5 \%$ of them, indicating that the cells were growing and surviving well in the hydrogel, and the hydrogel had good biocompatibility.

3. Establishment of a rabbit hindlimb ischemia model and stem cell transplantation experiment 3.1 Establishment of a rabbit hindlimb ischemia model and angiography

To generate a rabbit hindlimb ischemia model, the external iliac artery, femoral artery, and related branches were ligated during the operation, and the femoral artery segment from the external iliac artery to below the knee was removed (Fig. 3-1A, 1B). The skin color and skin temperature of the bilateral hindlimbs were compared. The skin color of the operated hindlimb was obviously darker than on the healthy side, the skin temperature of the operated hindlimb was significantly lower than that of the contralateral side, and the arterial pulsation could not be felt on the operated side (Fig. 3-1C). Through hindlimb angiography, no blood flow through the main blood vessels or their branches was observed in the hindlimb on the operated side (Fig. 3-1D).

3.2 General clinical evaluation after stem cell transplantation

Skin temperature data (difference in skin temperature between the operated hindlimb and the healthy hindlimb) were used for analysis. After 30 days of treatment, the difference in skin temperature between the bilateral hindlimbs of rabbits was $0.40 \pm 0.23^{\circ} \mathrm{C}$ in group $\mathrm{A}, 0.48 \pm 0.21^{\circ} \mathrm{C}$ in group $\mathrm{B}$, and $0.53 \pm$ $0.23{ }^{\circ} \mathrm{C}$ in group C. Pairwise comparisons in analysis of variance (ANOVA) showed that the skin temperature differences of the hindlimbs did not differ significantly between the three groups $(P>0.05)$ (Fig. 3-3A).

\subsection{Histological analysis}

The rabbit hindlimb skeletal muscle specimens were HE-stained. The hindlimb skeletal muscle fibers on the operated side of rabbits in group A (hydrogel + stem cell group) were arranged fairly neatly, there were fewer instances of vasculature disruption and disorganization, and there were mild disturbances of muscle fibers compared to fibers in normal tissue sections. Tissue sections of rabbits in group B (simple stem cell group) showed that the distribution of the vasculature and the arrangement of muscle fibers were not as good as in group A, but there was no obvious muscle fiber dissolution or necrosis. Tissue sections from group C (negative control group) showed obvious tissue structural disorders, partial dissolution and necrosis of muscle fibers, vascular system disorders, and extensive fibrosis (Fig. 3-2. $A H)$.

Histological scores after HE staining were $70.33 \pm 6.40$ in group $A, 66.00 \pm 5.41$ in group $B$, and $58.00 \pm$ 6.21 in group $C$, (Fig. 3-3B). The three groups of data were compared pairwise using ANOVA. The differences between the $B$ and $C$ groups and the $A$ and $C$ groups were statistically significant $(P<0.01)$, while the difference between the $A$ and $B$ groups was not significant $(P=0.055)$.

After performing immunofluorescence staining for factor VIII, we counted cells with positive immunofluorescent staining, and the results were $287.83 \pm 39.33$ in group $A, 219.33 \pm 47.04$ in group $B$, 
$185.33 \pm 41.70$ in group C, and $434.33 \pm 44.99$ in the normal group (the healthy hind limb) (Fig. 3- 3C). The four groups of data were analyzed by pairwise analysis with ANOVA, and the intergroup differences between the four groups of data were all statistically significant. Specifically, the differences between the three groups $A, B$, and $C$ and the normal group were statistically significant $(P<0.01)$, the difference between groups $A$ and $C$ was statistically significant $(P<0.01)$, and the differences between groups $A$ and $B$ and between groups $B$ and $C$ were also statistically significant $(P<0.05)$.

\section{Discussion}

At present, research on the application of stem cells to clinical diseases is very popular. Some researchers have transplanted stem cells into the body to treat a variety of diseases and have achieved certain therapeutic effects. For example, stem cells have been used in fields such as plastic surgery and cardiocerebrovascular diseases, providing strong support for the clinical application of stem cells ${ }^{[13-17]}$. As a new type of adult stem cells, AD-MSCs can meet the standards of ideal seed cells in tissue engineering and have incomparable advantages in tissue engineering technology or clinical applications.

According to the experimental results of this study, AD-MSCs derived from primary culture and expansion have biological characteristics of other adult stem cells. In terms of morphology, they exhibit fibroblastlike, spindle-shaped, and school-of-fish-like morphology, consistent with the morphological characteristics of mesenchymal stem cells in previous studies. To conduct flow cytometry immunophenotyping of the isolated cells, we selected five immunophenotypes, CD29, CD31, CD34, CD44, and HLA-DR. CD31 was used to exclude the small amount of vascular endothelial cell contamination from adipose tissue in the acquired AD-MSCs. CD34 was used to exclude the contamination from small amounts of adherent cells in the residual blood. HLA-DR was used to exclude contamination of fibroblasts from adipose tissue fascia and connective tissue. Positive CD29 and CD44 expression were used to further confirm that the cells obtained in the study were AD-MSCs.

In addition, the obtained stem cells were subjected to cell growth curve and cell cycle measurements and presented features consistent with the growth stages of stem cells, that is, the growth latency period, exponential growth period, and plateau period in which growth was inhibited. The growth curve was approximately a standard S-shape, consistent with the growth characteristics of AD-MSCs, further supporting the characterization of the obtained cells from another perspective.

AD-MSCs also have multi-directional differentiation potential. They can differentiate into different types of cells, such as osteoblasts, cardiomyocytes, adipocytes, endothelial cells, and chondrocytes, playing corresponding clinical roles. We investigated the osteogenic, adipogenic, and endothelial differentiation of obtained AD-MSCs, AD-MSCs could be induced into osteogenic, adipogenic, and endothelial differentiation under corresponding induction conditions.

In 1993, Professor Zhang Shuguang et al. ${ }^{[18]}$ discovered self-assembling ionic complementary polypeptides and synthesized a hydrogel using the polypeptides. The hydrogel scaffold is composed of a 
nanofiber network with uniform pores and rich water content (more than 99\%). The structure is similar to the extracellular matrix and can well mimic the internal environment to conduct three-dimensional culture of cells.

This study demonstrated that RAD/KLT/RGD, a functionalized self-assembling nanopeptide hydrogel, can promote both cell adhesion and such stem cell biological characteristics as proliferation and migration. In terms of its functionalized activity, after inoculation with AD-MSCs and under the culture conditions of EGM2-MV $+40 \mathrm{ng} / \mathrm{ml}$ VEGF $+10 \mathrm{ng} / \mathrm{ml}$ b-FGF, three-dimensionally cultured AD-MSCs stretched well, were connected to each other, and presented the morphological trend of forming a lumen. AO/EB staining showed that the three-dimensionally cultured cells survived well, with fewer than $5 \%$ apoptotic cells, and that the functionalized self-assembled nanopeptide hydrogel could allow a larger number of cells to show adherent growth within a certain time. Therefore, it has good application prospects in the field of vascular tissue engineering as a three-dimensional material for carrying stem cells ${ }^{[19.20]}$.

For some ischemic clinical diseases, the generation of new blood vessels in the ischemic tissue determines the survival and functional retention of the tissue. Therefore, it will be valuable to construct blood vessels and promote neovascularization in tissue engineering technology. At present, stem cell transplantation has become an emerging solution to promote blood vessel vascularization.

Some researchers believe that the transplanted stem cells can play a role by differentiating into endothelial cells, and the newly generated endothelial cells can form capillaries or participate in the neovascularization of some collateral vessels, thereby increasing the blood supply to the ischemic site. Other studies have suggested that transplanted stem cells can secrete a variety of angiogenic cytokines, including vascular endothelial growth factor (VEGF), fibroblast growth factor (FGF), hepatocyte growth factor (HGF), and insulin-like growth factor 1 (IGF-1), that act via paracrine mechanisms, thereby promoting angiogenesis and sprouting, increasing blood perfusion, and achieving the goal of improving ischemia ${ }^{[21-25]}$.

The transplantation of biomaterials combined with the delivery of stem cells to the ischemic site may be able to buffer the sudden changes in the living environment of stem cells. In addition, the growth, proliferation, and migration of stem cells in a three-dimensional culture scaffold could achieve a sustained release and long-lasting effect for the treatment of ischemic tissue. Therefore, using a biomaterial scaffold loaded with stem cells for a combination transplantation of stem cells and bioscaffold may achieve a better therapeutic effect than the traditional method of stem cell suspension injection. Based on the above ideas, this study used a functionalized self-assembled nanopeptide hydrogel combined with AD-MSCs to replace the previous method of stem cell suspension injection, aiming to improve the therapeutic effect of transplanted stem cells on ischemic tissue.

\section{Conclusion}


This study explored the transplantation of functionalized self-assembling peptide hydrogel-loaded ADMSCs into the ischemic hindlimb of rabbits and applied methods such as tissue section scoring and immunostaining to count positively stained cells to objectively evaluate the treatment effect. The combination transplantation had a better therapeutic effect than stem cell suspension injection alone, confirming that functionalized self-assembling nanopeptide hydrogels combined with AD-MSCs can promote vascular regeneration.

\section{Abbreviations}

\begin{tabular}{|ll|}
\hline Ad-MSCs & adipose-derived mesenchymal stem cells \\
\hline AFM & Atomic force microscopy \\
\hline AO/EB & Acridine orange ethidium bromide \\
\hline BMSCs & Bone marrow mesenchymal stem cells \\
\hline b-FGF & Basic fibroblast growth factor \\
\hline DMEM & Dulbecco's modified eagle's medium \\
\hline DMSO & Dimethyl sulfoxide \\
\hline DSA & Digital subtraction angiography \\
\hline EDTA & Eathylene diamine tetraacetic acid \\
\hline EGM2 & Endothelial cell growth medium-2 \\
\hline FBS & Fetal bovine serum \\
\hline H\&E & Hematoxylin-eosin staining \\
\hline HGF & Hepatocyte growth factor \\
\hline IGF & Insulin-like growth factor \\
\hline KLT & Functionalized peptide contained the VEGF mimicking sequence \\
\hline O.D & Optical density \\
\hline PBS & Phosphate buffered saline \\
\hline RADA & Self-assembling peptide \\
\hline SEM & Scanning electron microscope \\
\hline VEGF & Vascular endothelial growth factor \\
\hline 3D & 3-Dimensional \\
\hline
\end{tabular}

\section{Declarations}




\section{Competing interests}

The authors declare that they have no competing interests.

\section{Ethics approval and consent to participate}

All animal experiments have been approved by the Research Ethics Committee of Shandong University \Approval number》 KYLL-2015(KS)-200囚 and National Institutes of Health (NIH) Guidelines for the Care and Use of Laboratory.

\section{Funding}

This study was supported by grants from the Natural Science Foundation of Shandong Province (ZR2017MH072); the Natural Science Foundation Program of Shandong Province (2016GSF201219); and Livelihood Technology Project of Qingdao(18-6-1-90-nsh ).

\section{Consent for publication}

All authors of this manuscript agreed to publication.

\section{Acknowledgements}

The authors would like to express their gratitude to Dr. Jianmin Ling for their assistance in performing the experiments.

\section{Data Availability Statement}

The data that support the findings of this study are available from the corresponding author upon reasonable request.

\section{Authors' contributions}

Zhanao Liu performed the experiments, analyzed and interpreted the data, and wrote the manuscript. Tian Ailing, Xin Yi, Fan Lufeng; Shao Wenchong and Wu Han performed the analysis and interpretation of the data. Sun Nianfeng designed the study and revised the manuscript. All authors read and approved the final manuscript.

\section{References}

1. Bowen JE. Technical issues in harvesting and concentrating stem cells (bone marrow and adipose). PM R. 2015;7(4 Suppl):8-18.

2. Dodson MV, Wei S, Duarte M, et al. Cell supermarket: adipose tissue as a source of stem cells. J Genomics. 2013;1:39-44. 
3. Zuk PA, Zhu M, Ashjian P, et al. Human adipose tissue is a source of multipotent stem cells. Mol Biol Cell. 2002;13(12):4279-95.

4. Pittenger MF, Mackay AM, Beck SC, et al. Multilineage potential of adult human mesenchymal stem cells. Science. 1999;284(5411):143-7.

5. Shi L, Yang X. [Differentiation potential and application of stem cells from adipose tissue]. Zhongguo Xiu Fu Chong Jian Wai Ke Za Zhi. 2012;26(8):1007-11.

6. Zhu X, Du J, Liu G. The comparison of multilineage differentiation of bone marrow and adiposederived mesenchymal stem cells. Clin Lab. 2012;58(9-10):897-903.

7. Drury JL, Mooney DJ. Hydrogels for tissue engineering: scaffold design variables and applications. Biomaterials. 2003;24(24):4337-51.

8. Wang W, Wang W, Jiang Y, et al. Human adipose-derived stem cells modified by HIF-1a accelerate the recovery of cisplatin-induced acute renal injury in vitro. Biotechnol Lett. 2014;36(3):667-76.

9. Povsic TJ. Current State of Stem Cell Therapy for Ischemic Heart Disease. Curr Cardiol Rep. 2016;18(2):17.

10. Xie N, Li Z, Adesanya TM, et al. Transplantation of placenta-derived mesenchymal stem cells enhances angiogenesis after ischemic limb injury in mice. J Cell Mol Med. 2016;20(1):29-37.

11. Hou L, Kim JJ, Woo YJ, Huang NF. Stem cell-based therapies to promote angiogenesis in ischemic cardiovascular disease. Am J Physiol Heart Circ Physiol. 2016;310(4):H455-65.

12. Merino-González C, Zuñiga FA, Escudero C, et al. Mesenchymal Stem Cell-Derived Extracellular Vesicles Promote Angiogenesis: Potencial Clinical Application. Front Physiol. 2016;7:24.

13. Suzuki E, Fujita D, Takahashi M, Oba S, Nishimatsu H. Adipose tissue-derived stem cells as a therapeutic tool for cardiovascular disease. World J Cardiol. 2015;7(8):454-65.

14. Yang D, Wang ZQ, Deng JQ, et al. Adipose-derived stem cells: A candidate for liver regeneration. J Dig Dis. 2015;16(9):489-98.

15. Kingham PJ, Reid AJ, Wiberg M. Adipose-derived stem cells for nerve repair: hype or reality. Cells Tissues Organs. 2014;200(1):23-30.

16. Yeh DC, Chan TM, Harn HJ, et al. Adipose tissue-derived stem cells in neural regenerative medicine. Cell Transplant. 2015;24(3):487-92.

17. Bielli A, Scioli MG, Gentile P, Cervelli V, Orlandi A. Adipose Tissue-Derived Stem Cell Therapy for PostSurgical Breast Reconstruction-More Light than Shadows. Adv Clin Exp Med. 2015;24(3):545-8.

18. Zuk PA, Zhu M, Mizuno H, et al. Multilineage cells from human adipose tissue: implications for cellbased therapies. Tissue Eng. 2001;7(2):211-28.

19. Dodson MV, Wei S, Duarte M, et al. Cell supermarket: adipose tissue as a source of stem cells. J Genomics. 2013;1:39-44.

20. Rosa S, Praqa C, Pitrez PR, Gouveia PJ, Aranguren XL, Ricotti L. Ferreira L.S. Functional characterization of iPSC-derived arterial- and venous-like endothelial cells. Sci Rep. 2019;9:3826-32. 
21. Sellke FW, Laham RJ, Edelman ER, Pearlman JD, Simons M. Therapeutic angiogenesis with basic fibroblast growth factor: technique and early results. Ann Thorac Surg. 1998;65(6):1540-4.

22. Aoki M, Morishita R, Taniyama $Y$, et al. Angiogenesis induced by hepatocyte growth factor in noninfarcted myocardium and infarcted myocardium: upregulation of essential transcription factor for angiogenesis, ets. Gene Ther. 2000;7(5):417-27.

23. Kamihata $\mathrm{H}$, Matsubara $\mathrm{H}$, Nishiue $\mathrm{T}$, et al. Implantation of bone marrow mononuclear cells into ischemic myocardium enhances collateral perfusion and regional function via side supply of angioblasts, angiogenic ligands, and cytokines. Circulation. 2001;104(9):1046-52.

24. Wafai R, Tudor EM, Angus JA, Wright CE. Vascular effects of FGF-2 and VEGF-B in rabbits with bilateral hind limb ischemia. J Vasc Res. 2009;46(1):45-54.

25. Salvolini E, Orciani M, Vignini A, Mattioli-Belmonte M, Mazzanti L, Di PR. Skin-derived mesenchymal stem cells (S-MSCs) induce endothelial cell activation by paracrine mechanisms. Exp Dermatol. 2010;19(9):848-50.

\section{Figures}



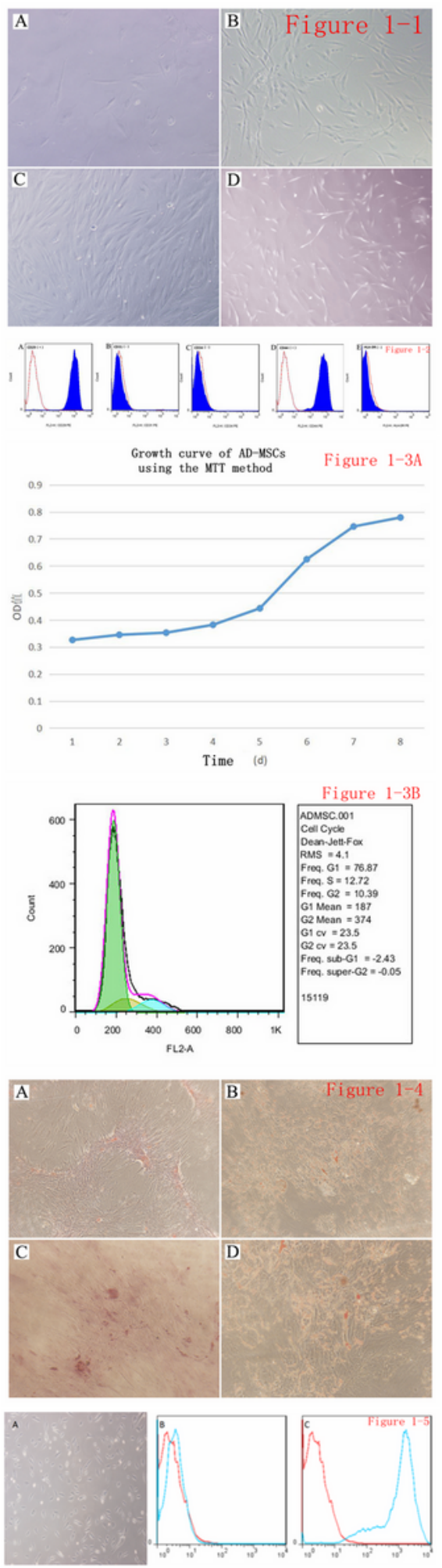

\section{Figure 1}

Morphological appearance of AD-MSCs A: Adherent primary AD-MSCs $(\times 200)$. B: Primary AD-MSCs $(\times$ 100). C: P3 AD-MSCs (× 100). D: P10 AD-MSCs (× 100). Immunophenotyping of AD-MSCs by flow cytometry A: Positive expression of CD29. B: Negative expression of endothelial cell marker CD31. C: Negative expression of hematopoietic marker CD34. D: Positive expression of CD44. E: Negative expression of fibroblast marker HLA-DR. Growth curve of AD-MSCs using the MTT method After cells 
were treated with MTT solution, the cell growth curve was drawn using the OD values (mean) measured over 8 days. The growth of AD-MSCs went through a latency phase, exponential growth phase, and plateau phase, making an S-shaped curve. Growth cycle determination of AD-MSCs Induction of osteogenic and adipogenic differentiation of AD-MSCs A, C: Induction of AD-MSCs to differentiate into osteoblasts. Alizarin red S staining shows calcium nodule deposition in induced AD-MSCs, A ( $\times 100), C(\times$ 200). B, D: Induction of AD-MSCs to differentiate into adipocytes. Oil red $O$ staining shows lipid droplet accumulation in the induced AD-MSCs, B $(\times 100), D(\times 200)$. Induction of endothelial differentiation of AD-MSCs and identification of immunophenotypes by flow cytometry A: AD-MSCs in the 3D-printed model were induced to differentiate, and paving stone-like changes were seen in the cells $(\times 100)$. B: Negative expression of CD31 in the control group. C: Positive expression of CD31 in the experimental group. 

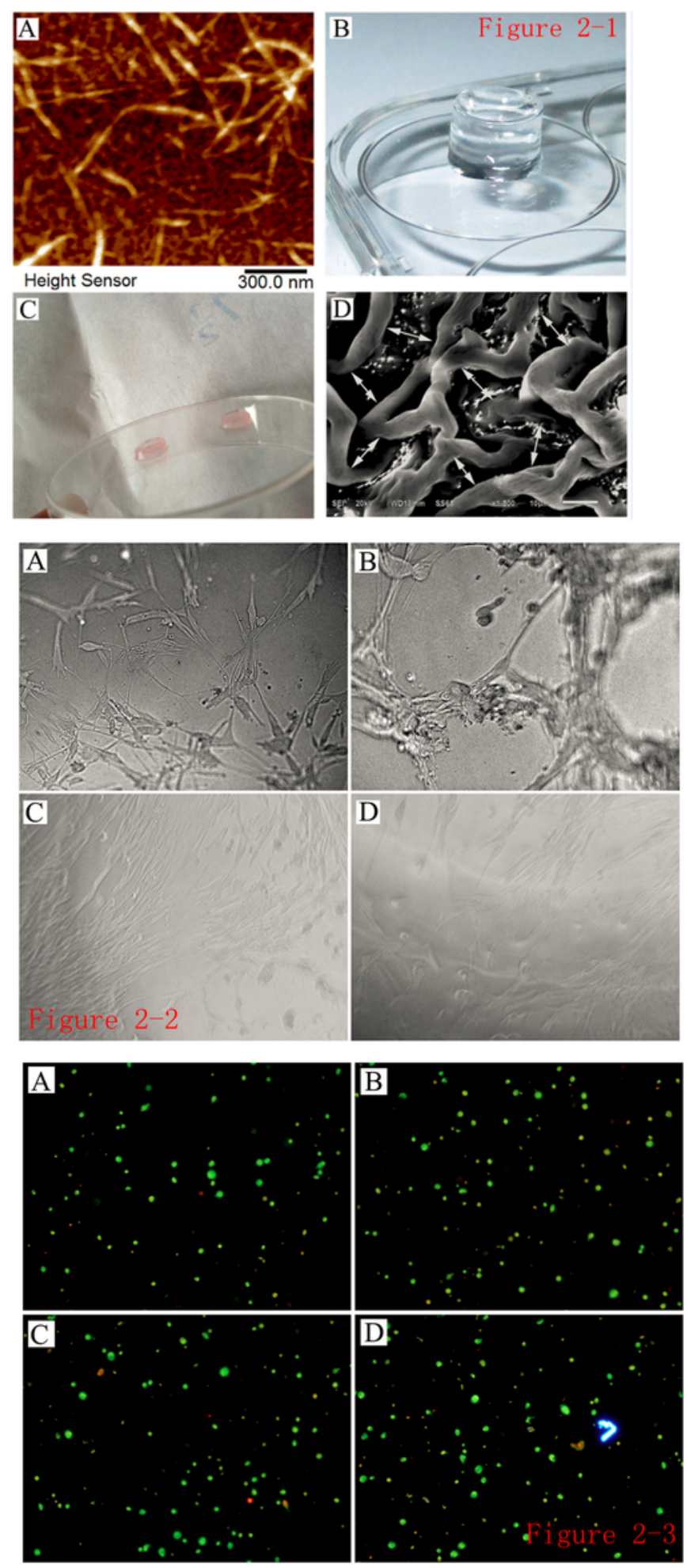

\section{Figure 2}

Atomic-force microscopy detection of peptides and scanning electron microscopy detection of peptide hydrogels A: Atomic-force microscopy inspection of the prepared samples of the peptide solution, revealing scattered nanofibers with uniform fiber thickness and length $(\times 30,000)$. B: Properties of the self-assembled hydrogel in the Transwell chamber. The assembled hydrogel was transparent and rich in water content. C: The hydrogel took on the pale-red color of the culture medium after the components of 
the hydrogel were neutralized by the culture medium of AD-MSCs. D: The pore-like, uniform internal structure of the hydrogel under the scanning electron microscope $(\times 500)$. Observation of cells inside the self-assembled nanopeptide hydrogel model combined with AD-MSCs A, B, C, D: Images of cells in the hydrogel photographed at different views, showing that the cells in the hydrogel grew well and adhered tightly $(\times 100)$. AO/EB staining image of cell suspension after culturing AD-MSCs in the hydrogel The obtained AD-MSC suspension was subjected to AO/EB staining, the cell debris was removed, and cell counting revealed red cells made up $<5 \%$.
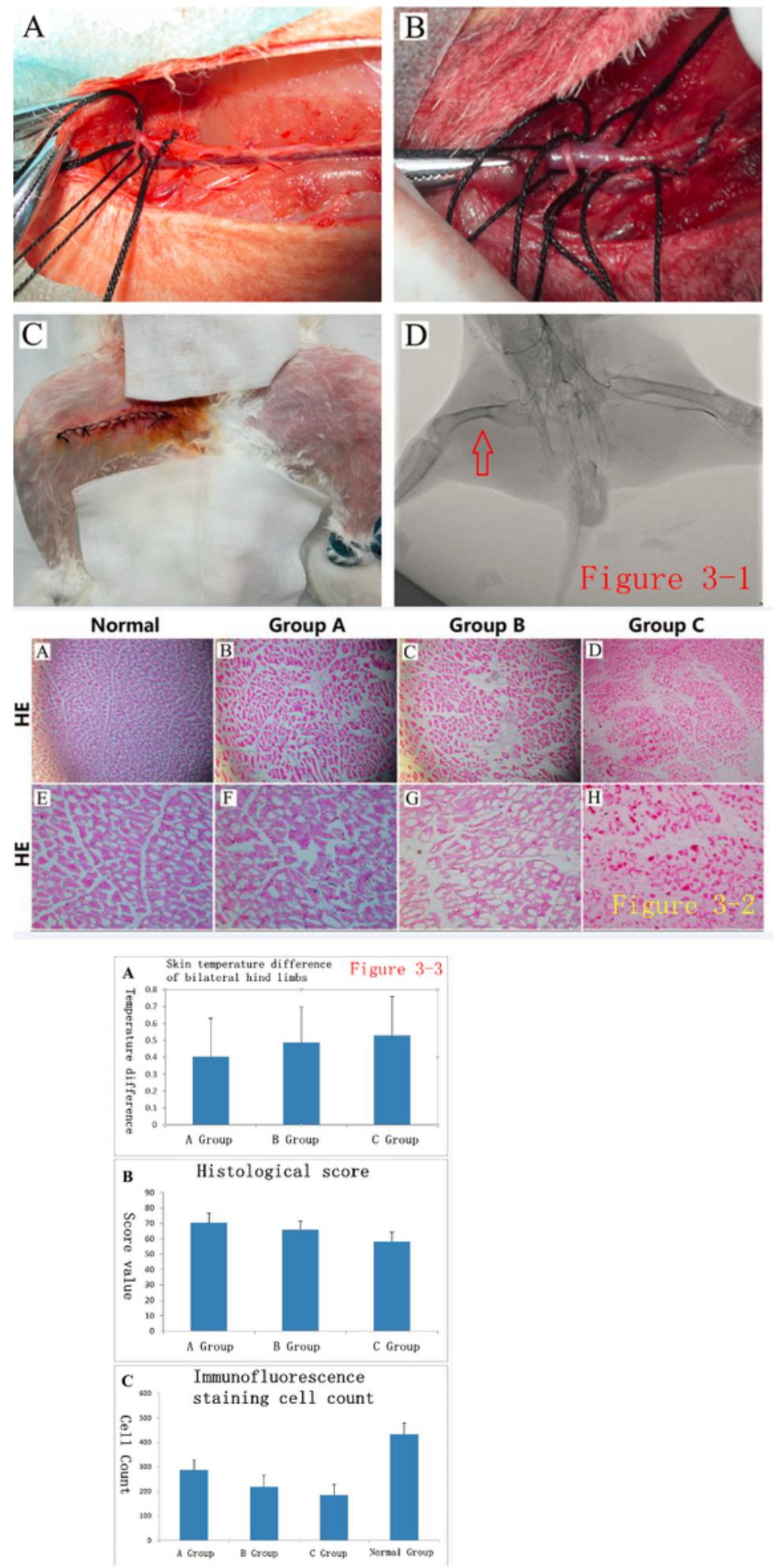


\section{Figure 3}

Generation of a rabbit hindlimb ischemia model A, B: The model of rabbit hindlimb ischemia was generated by ligating and excising the segment of femoral artery from the end of the external iliac artery to below the knee. C: The skin color of the hindlimbs was compared after the rabbit hindlimb ischemia model was made. D: Digital subtraction angiography showed no arterial arteriography in the main arteries of the operated hindlimb. Histological evaluation of the rabbit model of hindlimb ischemia Observation of HE-stained rabbit hindlimb skeletal muscle specimens revealed that the skeletal muscle fibers of the operated hindlimb in group A (hydrogel + stem cell group) were arranged fairly neatly, and there were few instances of vasculature disruption and disorganization. Group B (simple stem cell group) showed that the distribution of the vasculature and the arrangement of muscle fibers were not as good as those of group A, but there were no obvious muscle fiber dissolution and necrosis. Group C (negative control group) showed obvious tissue-structural disorders, partial dissolution and necrosis of muscle fibers, and vascular system disorders. Statistical analysis of general data and histological data of rabbits after surgery A. Thirty days after treatment, the skin temperature differences of bilateral hindlimbs in rabbits were $0.40 \pm 0.23$ in group $A, 0.48 \pm 0.21$ in group $B$, and $0.53 \pm 0.23$ in group $C$. B. Histological scores after $\mathrm{HE}$ staining were $70.33 \pm 6.40$ in group $A, 66.00 \pm 5.41$ in group $B$, and $58.00 \pm 6.21$ in group C. C. After immunofluorescence staining with factor VIII, the numbers of immunofluorescence-positive cells were counted: $287.83 \pm 39.33$ in group $A, 219.33 \pm 47.04$ in group $B, 185.33 \pm 41.70$ in group $C$, and $434.33 \pm 44.99$ in the normal group (the healthy-side hind limbs)

\section{Supplementary Files}

This is a list of supplementary files associated with this preprint. Click to download.

- Arrivechecklist.docx 